\title{
Multiscale mechanics of biological, bioinspired, and biomedical materials
}

\author{
Christian Hellmich and Dinesh Katti, Guest Editors
}

\begin{abstract}
Mechanical property measurement protocols have their origins in metallurgy as well as in mechanical and civil engineering-metals being the first materials used on a broad industrial scale. Recent decades have evidenced growing interest in applying these protocols to biological materials or materials mimicking or replacing biological tissue. However, the mechanical properties of biological materials have been found to be highly variable and seemingly hard to capture by traditional protocols. A slow, emerging thought is that perhaps the mechanical theories underlying the testing protocols emanating from the metals field might not be fully applicable to the highly complex, hierarchically organized biological materials and might need further development. The articles in this issue highlight different and complementary, yet also interdependent, approaches to the challenge of extending theoretical and applied mechanics to the level needed for satisfying and reliably capturing the properties of biological materials. This issue also encompasses corresponding, far-reaching consequences for measurement techniques and evaluation protocols aimed at the determination of mechanical protocols.
\end{abstract}

\begin{abstract}
From measuring mechanical properties to a novel mechanics science of biological materials

Measuring mechanical properties is an integral part of almost any characterization procedure in state-of-the-art materials science. Such measurements are typically done according to standardized protocols, ${ }^{1,2}$ both for equipment characteristics and data evaluation. These protocols have their origins in metallurgy, as well as mechanical and civil engineeringmetals being the first materials used on a broad industrial scale. Over the course of time, the same or similar protocols were used for other materials, and recent decades have evidenced a growing interest in applying them to either biological materials or materials mimicking or replacing biological tissue.
\end{abstract}

An immediate outcome of these activities was that the mechanical properties of biological materials were found to be highly variable and difficult to capture by the aforementioned protocols. One thought was that the mechanical theories underlying the testing protocols emanating from the metals field might not be fully applicable to the highly complex, hierarchically organized biological materials and might need further development, and the corresponding basic assumptions may need to be rethought and improved. These developments, driven by the applied physics and chemistry communities, as well as the mechanical and civil engineering communities, have left their traces in these various scientific fields and reflected back on materials science at large. This is illustrated by the various and ever-growing symposia at Materials Research Society (MRS) Meetings and other conferences over the last decade.

This issue of MRS Bulletin is a compilation of articles highlighting different, complementary, and interdependent approaches to the challenge of extending theoretical and applied mechanics to the level needed for reliably capturing the properties of biological materials. The articles also include analogous, extensive consequences for measurement methods and evaluation protocols intended to determine mechanical procedures.

\section{Improving evaluation protocols: From} stress-strain curves to elaborate back-analysis schemes founded on mechanical principles

Problems with proper determination of elastic properties of biological materials and biomaterials might start with basic and seemingly well-understood quantities such as Young's modulus. As one standard measure of elastic material behavior, Young's modulus can be determined from the linear portion of loading in a load-displacement curve obtained from quasistatic tests; this is in case the tested material exhibits a pronounced purely elastic regime, as is normally encountered

Christian Hellmich, Institute for Mechanics of Materials and Structures, Vienna University of Technology (Tu Wien), Austria; christian.hellmich@tuwien.ac.at Dinesh Katti, Department of Civil and Environmental Engineering, North Dakota State University, USA; dinesh.katti@ndsu.edu

DOI: $10.1557 / \mathrm{mrs} .2015 .65$ 
with metals. This evaluation rule is often applied to biological materials and biomaterials as well. However, these may show inelastic behavior even at very low load levels, so that the aforementioned rule applies to an elastoplastic, rather than an elastic modulus. The remedy to this can be obtained by reviewing the thermodynamic definition of elasticity (i.e., the stress-driven deformation related to energy that can be fully recovered as efficient mechanical work). ${ }^{3}$

Consequently, it is the unloading phase of a quasistatic test where the mechanical work is provided by the material, and it is the slope of the unloading curve (coinciding with the loading curve in the case of metals, but not necessarily in the case of biomaterials) that delivers the elastic Young's modulus in a reliable fashion (Figure 1) ${ }^{4,5}$ It should be noted that for reliable evaluation of the elastic modulus of soils in civil engineering, the unloading slopes of the stress-strain curves are used. ${ }^{6}$ Although requiring caution, this is probably the simplest example of the need to consider the fundamentals of applied mechanics when determining mechanical properties.

In most biological materials, particularly in soft tissues, the situation is much more challenging, with mechanical properties strongly dependent on the deformation state, and with material functions rather than numbers being required to quantify mechanical properties. This implies that experimental measurements need to be regarded as the known "results" of sophisticated mechanical analysis, based on complex (but a priori unknown) stress-strain laws and non-trivial boundary conditions. The stress-strain material functions need to be back-analyzed from the experimental results by means of a challenging mathematical process called inverse analysis. In this issue, Morin and Avril review a particularly robust and efficient way to do this, namely using the virtual fields method and its application to arterial tissue. The complex stress-driven

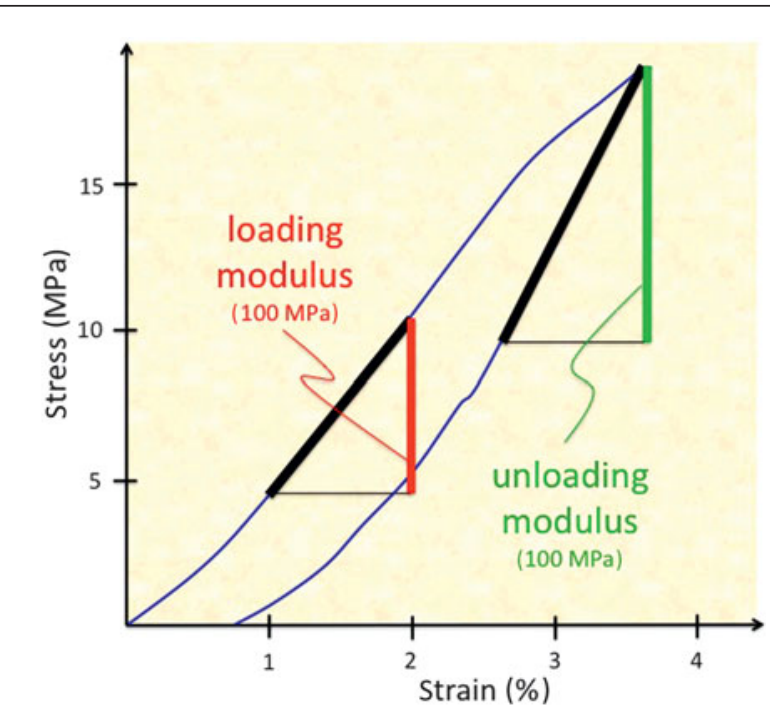

Figure 1. Loading and unloading moduli derived from the stress-strain curve of a polymer-ceramic-based biomedical material. $^{4}$ deformation behavior in this case stems from re-orientation of collagen and elastin fibers. This requires consideration of information at a level below that of macroscopic material testing; therefore, in addition to the complexities arising from inverse analysis, this represents an approach that may be classified as multiscale.

\section{Opening the door to the "inner life" of biological materials: Poromechanics coupled to transport and biology modeling}

Consideration of microstructural features in mechanical theories can be realized at different levels of sophistication, completion, or precision, typically dependent on the available experimental information and on the maturity of the employed mechanical theory. The second article, by Malandrino et al., describes a well-accepted route from established stress-strain laws to mathematical formulations that explicitly consider quantities at the microscopic scale, namely the volume fraction of the pore space (called porosity) found in biological tissues. This leads to the field called poromechanics, ${ }^{7}$ which has its origins in geotechnical engineering. Since this porosity is the location of important chemical and biological processes, it naturally provides a means to couple mechanical theories to physical chemistry, transport, and biology. In this context, a mechanical property reflects not only a complex stress-strain relation, but also the effects of interacting biological and chemical processes within the tissue. The article focuses on soft tissues such as vertebral discs. Poromechanics is also very beneficial for the understanding of hard tissues such as bone, in particular when combined with cell population kinetics, leading to a mechanobiological formulation that describes the process of bone remodeling by combining mechanics with systems biology (Figure 2). ${ }^{8,9}$

\section{Deciphering the role of the microstructure: Hierarchical approaches}

Although the aforementioned poromechanics approaches are already "informed" by important physical and biological processes below the "smeared-out" macroscopic scale of a representative volume element (RVE) comprising a statistically relevant amount of biological tissue, the experimental validation of such models is typically restricted to macroscopic observations. In other words, physical and mechanical properties of the intriguing micro- and nanocomponents of biological tissues are not explicitly introduced in theoretical formulations, and therefore, the corresponding experimental evidence stays largely unnoticed in the mathematical models rooted in classical poromechanics.

Overcoming this limitation through explicit consideration of elementary constituents of biological materials first gained attention in the realm of hard tissues, where rather soft (organic) components interact with rather hard (inorganic) components. Both together allow for an unusual and highly interesting coexistence of normally mutually exclusive mechanical properties such as high strength and toughness, a combination 


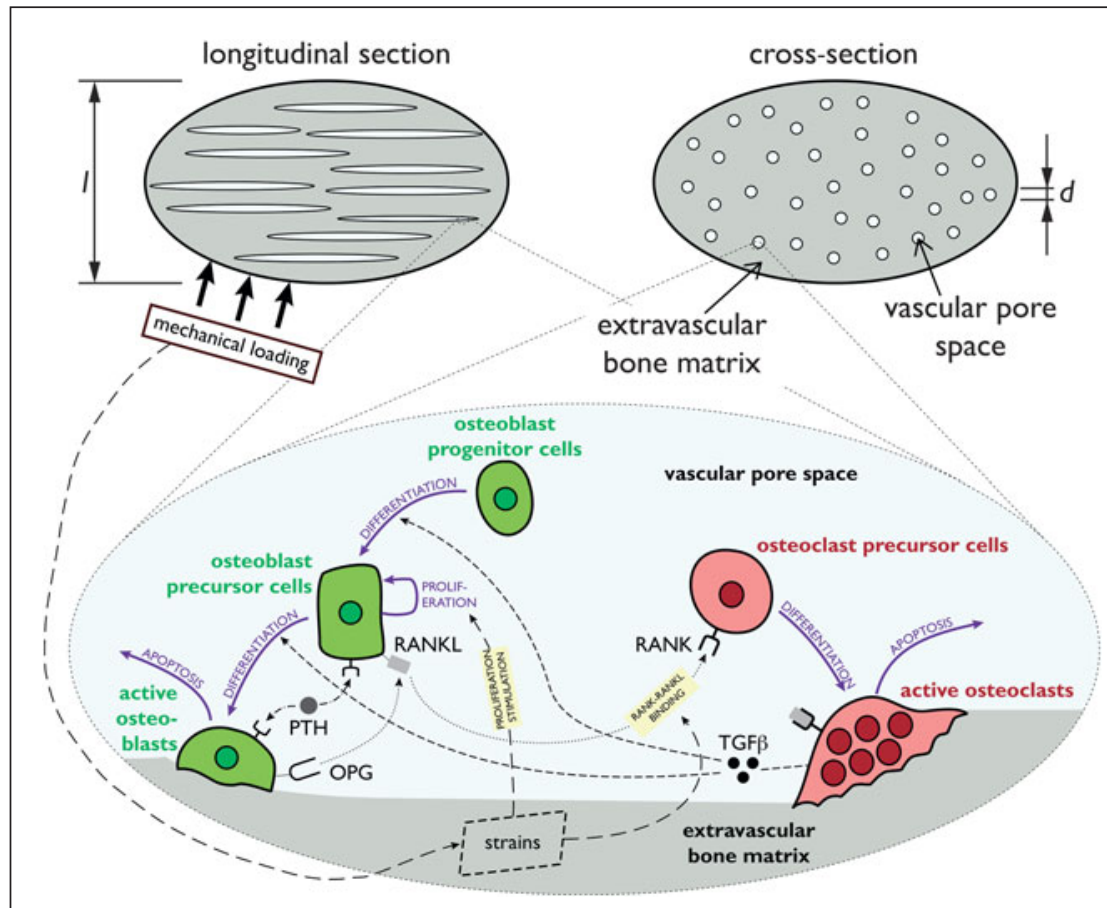

Figure 2. Poromechanics combined with systems biology: new modeling avenues for understanding the remodeling of bone. ${ }^{8,9}$ Pore spaces in bone are the location of important chemical and biological processes. Note: PTH, RANKL, OPG, RANK, and TGF $\beta$ are biochemical factors.

at the below-micron scale ${ }^{14}$ ). (2) For achieving satisfying micromechanical predictions of the overall material behavior of mineralized tissues such as bone, it needs to be considered that in contrast to an often popular assumption, the majority of mineral crystals lie outside the collagen fibrils. ${ }^{15}$ This was independently shown by pioneering, but often somewhat neglected, contributions using transmission electron microscopy ${ }^{16}$ and neutron diffraction techniques. ${ }^{17}$ (3) Interlocks between platelets appear to be key for the strength and toughness of nacre (see Figure 3). ${ }^{18}$ The aforementioned micromechanical approaches can be relatively straightforwardly extended to the realm of microporomechanics, ${ }^{19}$ considering also the effect of fluids filling different pore spaces, such as the intermolecular, the intercrystalline, the lacunar, and the vascular spaces in bone..$^{20,21}$ This also opens the hierarchical approaches described here to the coupled biochemo-mechanical analyses described in the previous section.

\section{Scrutiny into the nanostructure of elementary components: Molecular simulations}

never attained by traditional ceramic or metallic materials. The contribution by Okumura in this issue summarizes and reviews such peculiar interactions of hard and soft tissue components. He applies simple scaling laws, which indicate fundamental mechanisms behind amazing mechanical properties of material and structural systems such as nacre, crustacean exoskeletons, and spider webs.

Understanding of the aforementioned mechanisms promises to find routes for improving man-made materials through bioinspiration. At a more mature stage, this may be achieved by computer-aided material design, based on sophisticated computational strategies. The latter may typically originate from the well-established field of engineering mechanics, as it is broadly applied in civil and mechanical engineering. In fact, the last decade-and-a-half has experienced a surge of engineering mechanics-based approaches for deciphering the mechanical implications of the hierarchical organization of biological materials, including finite element analyses ${ }^{10}$ and continuum micromechanics or random homogenization strategies. ${ }^{11}$ Often, they have been more than precise elaborations of earlier proposed scaling mechanisms but have revealed novel fundamental features untraceable by earlier approaches.

Three prominent examples can be presented in this regard: (1) The stiffness of organic components in hard tissues has been shown to be much larger than generally anticipated ${ }^{12,13}$ (but in accordance with the elasticity determined from highfrequency Brillouin light scattering tests characterizing collagen
All the aforementioned approaches have dealt with mechanical properties, such as stiffness and strength, which are inherently linked to the concept of continuum mechanics: Material properties are related to RVEs hosting statistically distributed matter. This also holds true for the properties of even the smallest elementary components, which therefore need to be defined by RVEs, which are significantly larger ${ }^{22}$ than any inhomogeneities within these RVEs. In the case of nanocrystals or molecular collagen, these inhomogeneities are already related to single molecules. Hence, continuum mechanics emerges as a very versatile tool, valid for many orders of magnitude length scales, down to cases where the inhomogeneities reach molecular size. When searching for the origins of the mechanical properties of the elementary building blocks themselves, it has become common to develop molecular dynamics approaches.

In this context, the focus has first been on the retrieval of bulk properties. Studies ${ }^{23,24}$ have shown the effects of chemical details such as cross-links on stiffness, but also on the brittleness of collagen fibrils. Such important qualitative insight has influenced the perception of the mechanical behavior of hierarchical biological materials, such as bone through multiscale continuum approaches. Bone strength was understood to be the result of ductile sliding of mineral crystals followed by the brittle failure of collagen. ${ }^{25}$

Achieving quantitative reliability of results from molecular dynamics simulations seems to be a much trickier issue than previously thought and depends on rather surprising features. 


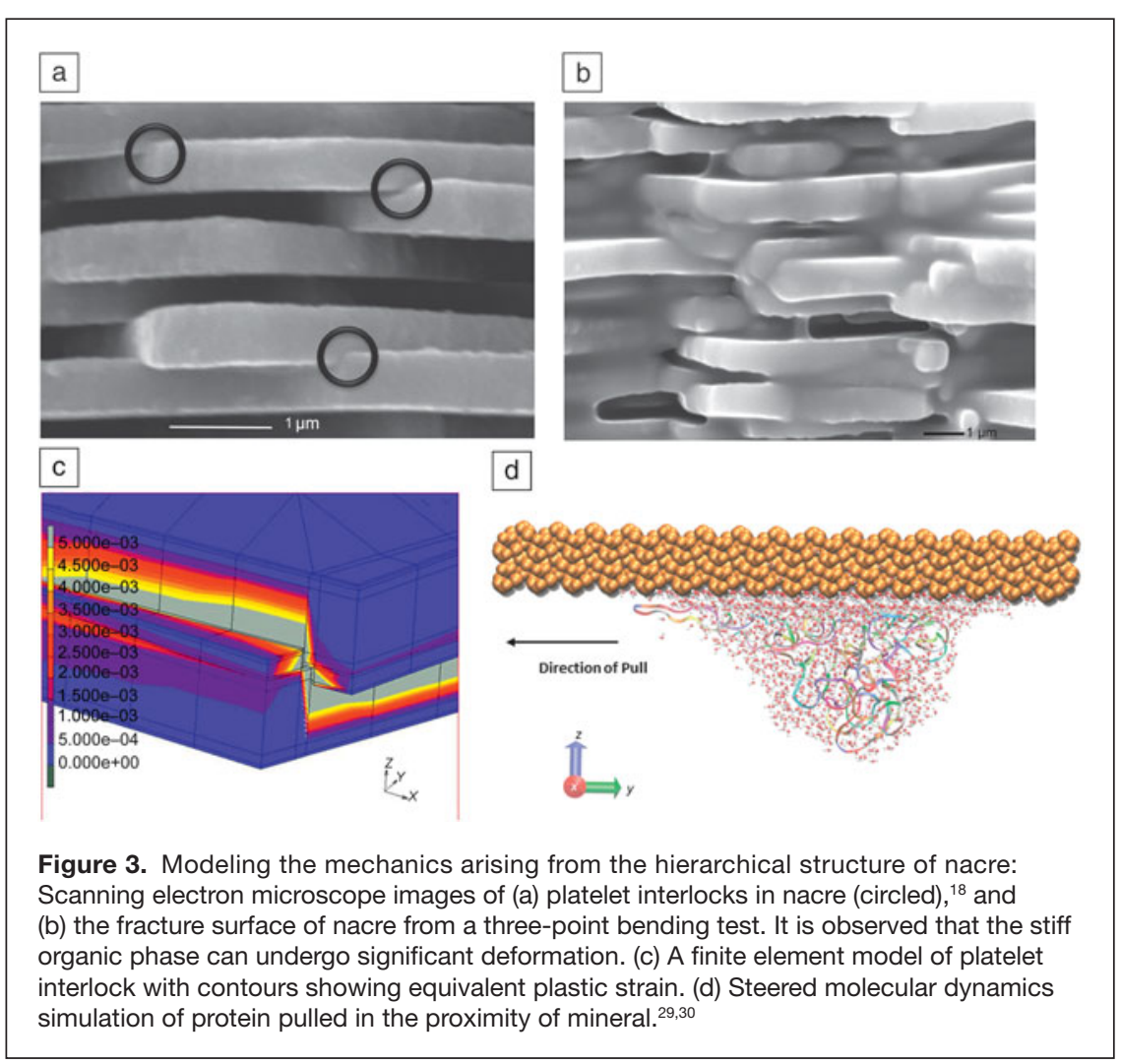

allows for realizing, one scale up, a large variability in mechanical properties with virtually the same underlying molecular elements.

\section{"Drowning in complexity" versus the quest for a unifying picture}

While molecular models may resolve features that are inaccessible to continuum models, their complexity, as a rule, dramatically restricts the size of the overall system to be simulated. This calls for a synergetic combination of molecular and continuum approaches. Keeping in mind the prominent role of interfaces as discussed before, recent achievements in continuum micromechanics have allowed for upscaling the properties of two-dimensional interfaces embedded in three-dimensional (3D) matrices to homogenized 3D material properties. ${ }^{28}$ The micro-interface properties, in turn, may then be accessible through molecular dynamics approaches focusing strongly on the interface problem, and leaving aside most of the 3D bulk problem. Qu et al. outline such an approach in the final contribution in this issue. It opens the perspective for a seamless suite of mechanical interaction pattern recognition, from the molecular scale up to the macroscopic scale.

For instance, to obtain more realistic stiffness properties of collagen molecules, very long chains rather than short sequences of this macromolecule need to be modeled (see Figure 4). ${ }^{26}$ Later on, the focus of molecular studies shifted from bulk to interface properties, revealing that the bonding type at interfaces between different fundamental building blocks plays an essential role. ${ }^{27}$ Leaving animal tissue systems aside for the moment and turning toward cellulose - the key ingredient of the vast majority of plants on our planet- the contribution of Sinko et al. in this issue explores how the unique nanostructure of cellulose nanocrystals provides the basis for a multitude of different interface types to neighboring constituents, which

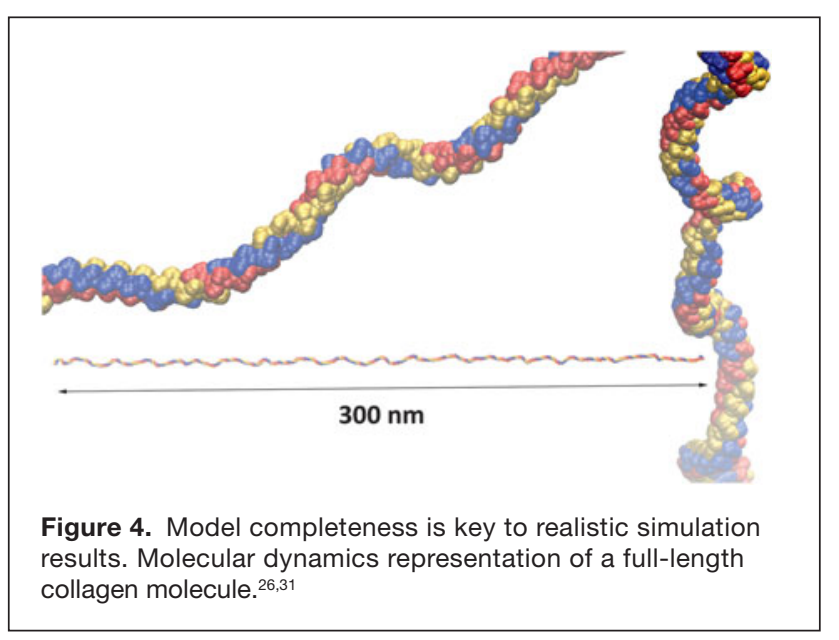

Such a numerical tool seems particularly interesting for deciphering creep and strength properties of biological materials, and as a means to mimic them in bioinspired engineering materials, or for patient-optimized implants.

\section{Summary}

Multiscale mechanics of biological materials is an extremely fascinating field integrating many aspects of materials science, physics, chemistry, and biology into a coherent, theoretically founded whole. It allows for deeper revelation of intriguing concepts nature employs for arriving at desirable (but often unique) combinations of beneficial mechanical properties (such as strength and toughness). This paves the way to more applications in materials design for mechanical, civil, and biomedical engineering. On the other hand, the multiscale mechanics field also strongly reflects back to the traditional communities of molecular physics and engineering mechanics, providing these communities with new experimental, theoretical, and computational tools for dealing with complexities which, as a rule, have not been encountered, expected, or investigated in the realm of traditional engineering materials.

\section{References}

1. ASTM, Standard Test Method for Young's Modulus, Tangent Modulus, and Chord Modulus, Designation E111-82, Philadelphia, (1992) pp. 288-293.

2. C. Turner, D. Burr, Bone 14, 593 (1993).

3. J. Salencon, Handbook of Continuum Mechanics (Springer, New York, 2001).

4. K.W. Luczynski, T. Brynk, B. Ostrowska, W. Swieszkowski, R. Reihsner, C. Hellmich, J. Biomed. Mater. Res. 101A, 138 (2013). 
5. K. Luczysnki, A. Steiger-Thirsfeld, J. Bernardi, J. Eberhardsteiner, C. Hellmich, J. Mech. Behav. Biomed. Mater., doi:10.1016/jmbbm.2015.03.001 (2015).

6. P.Y. Hichler, ASCE J. Geotech. Eng. 122, 641 (1996).

7. 0. Coussy, Poromechanics (Wiley, New York, 2004).

8. S. Scheiner, P. Pivonka, C. Hellmich, Comput. Methods Appl. Mech. Eng. 254, 181 (2013)

9. S. Scheiner, P. Pivonka, D. Smith, C. Dunstan, C. Hellmich, Int. J. Numer. Methods Biomed. Eng. 30, 1 (2014).

10. K. Katti, D. Katti, Mater. Sci. Eng. C 26C, 1317 (2006).

11. A. Fritsch, C. Hellmich, J. Theor. Biol. 244, 597 (2007)

12. D. Katti, K. Katti, J. Sopp, M. Sarikaya, Comput. Theor. Polym. Sci. 11, 397 (2001).

13. C. Hellmich, J.-F. Barthélémy, L. Dormieux, Eur. J. Mech. A. Solids 23A, 783 (2004).

14. S. Cusack, A. Miller, J. Mol. Biol. 135, 39 (1979).

15. C. Hellmich, F.-J. Ulm, J. Eng. Mech. 128, 898 (2002).

16. S. Lees, K. Prostak, Connect. Tissue Res. 18, 41 (1988).
17. S. Lees, L. Bonar, H. Mook, Int. J. Biol. Macromol. 6, 321 (1984).

18. K. Katti, D. Katti, S. Pradhan, A. Bhosle, J. Mater. Res. 20, 1097 (2005).

19. L. Dormieux, D. Kondo, F.-J. Ulm, Microporomechanics (Wiley, New York, 2006).

20. C. Morin, C. Hellmich, Ultrasonics 54, 1251 (2014).

21. C. Hellmich, D. Celundova, F.-J. Ulm, J. Eng. Mech. 135, 382 (2009).

22. A. Zaoui, J. Eng. Mech. 128, 808 (2002).

23. M. Buehler, Proc. Natl. Acad. Sci. U.S.A. 103, 12285 (2006)

24. M. Buehler, J. Mech. Behav. Biomed. Mater. 1, 59 (2008).

25. A. Fritsch, C. Hellmich, L. Dormieux, J. Theor. Biol. 260, 230 (2009).

26. S. Pradhan, D. Katti, K. Katti, J. Nanomech. Micromech. 1, 104 (2011).

27. R. Bhowmik, K.S. Katti, D.R. Katti, Mater. Sci. 42, 8795 (2007).

28. M. Shahidi, B. Pichler, C. Hellmich, Eur. J. Mech. A. Solids 45A, 41 (2014).

29. P. Ghosh, D.R. Katti, K.S. Katti, Biomacromolecules 8, 851 (2007).

30. P. Ghosh, D.R. Katti, K.S. Katti, J. Nanomater. (2008), doi: 10.1155/ 2008/582973.

31. S.M. Pradhan, K.S. Katti, D.R. Katti, Biomacromolecules 13, 2562 (2012). $\square$
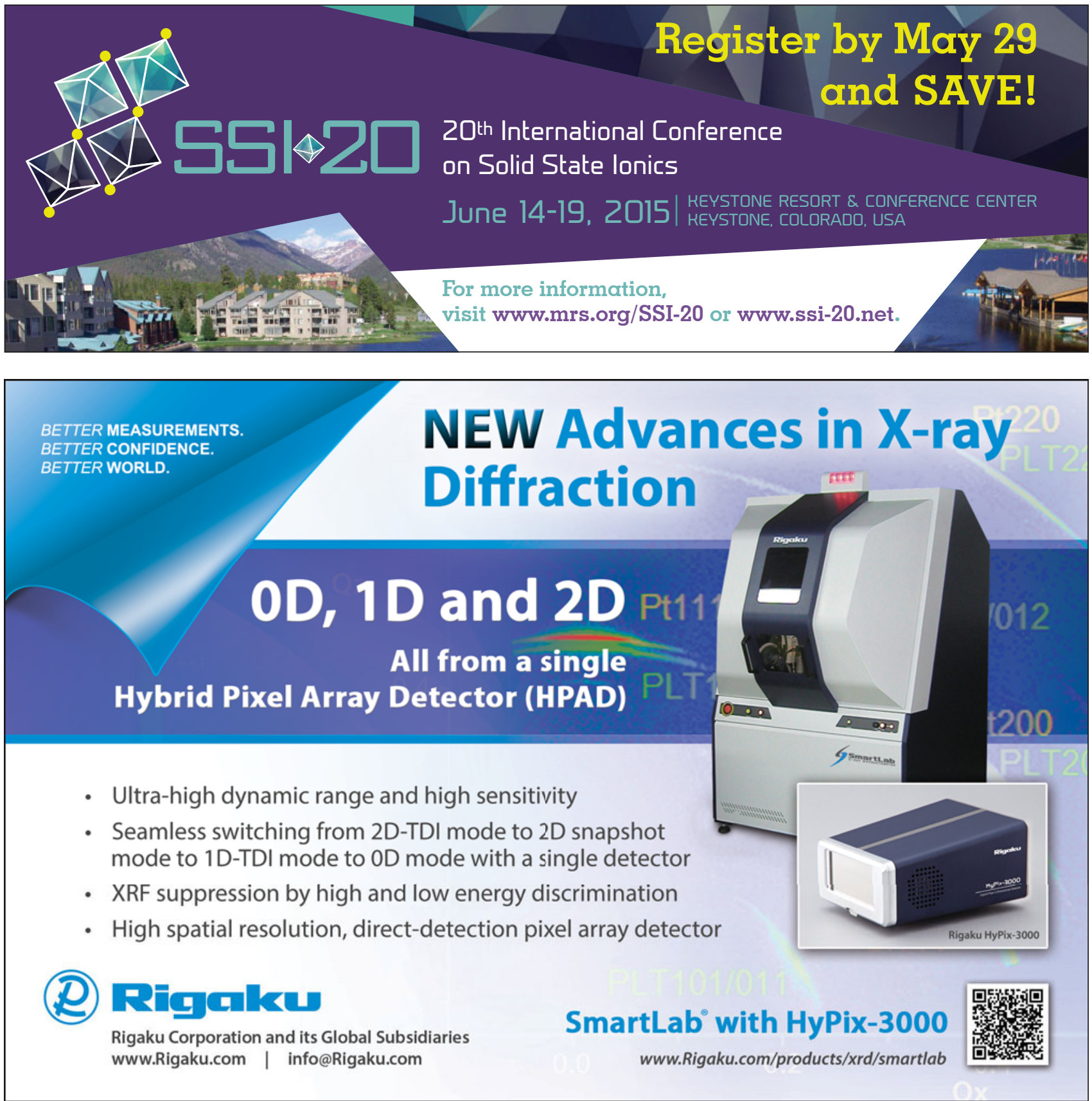